\title{
Simulación de los procesos de desoxigenación y reoxigenación en aguas contaminadas
}

\author{
Simulation of deoxygenation and reoxygenation processes in
}

polluted water

Recibido: julio 20 de 2020 | Revisado: agosto 26 de 2020 | Aceptado: setiembre 15 de 2020

\section{Luis Carrasco Venegas ${ }^{\mathrm{I}}$ \\ Luis Castañeda Perez ${ }^{2}$ JORGE LESCANO SANDOVAL ${ }^{3}$ \\ José Iannacone Oliver ${ }^{2}$}

1 Unidad de Investigación de la Facultad de Ingeniería Química, Facultad de Ingeniería Química, Universidad Nacional del Callao, Perú. lacarrascov@unac.edu.pe

2 Grupo de Investigación en Sostenibilidad Ambiental, Escuela Universitaria de Posgrado, Universidad Nacional Federico Villarreal.

3 Facultad de Ingeniería Geográfica, Ambiental y Ecoturismo, Universidad Nacional Federico Villarreal.

\begin{abstract}
Resumen
La simulación es una herramienta predictiva económica y rápida para conocer con antelación diversos procesos. En el presente trabajo, se ha aplicado a los procesos de desoxigenación y reoxigenación en los cuerpos de agua, por presencia de carga orgánica. Se ha modelado la demanda carbonácea y nitrogenada de los cuerpos de agua, tanto para régimen estacionario y no estacionario, considerando el modelo de advección, difusión y reacción. Para el caso no estacionario, se toman en cuenta dos escenarios: uno de ingreso instantáneo (tipo pulso) del contaminante y el otro, de ingreso permanente. La solubilidad del oxígeno en agua se obtiene mediante ecuaciones que consideran la influencia de la temperatura, el contenido de sal y la presión. Se desarrollaron, calibraron y ejecutaron los programas respectivos, considerando que las curvas de decaimiento de la materia orgánica, expresada en términos de la variación de la Demanda Bioquímica de Oxígeno (DBO) y el déficit de oxígeno (DO), tengan una evolución temporal y espacial tipo gaussiana. Como resultado se obtuvieron las curvas que describen el ingreso instantáneo del contaminante aguas abajo del punto de vertido, resultando ser tipo gaussianas y para el caso de ingreso permanente del contaminante, curvas hiperbólicas. Los modelos propuestos se utilizan para estimar el desplazamiento y los niveles de concentración de la carga orgánica; asimismo, el déficit critico de oxígeno del cuerpo de agua, parámetros importantes para evaluar la calidad del agua.
\end{abstract}

Palabras clave: modelamiento, simulación, cuerpo de agua, advección, difusión, reacción

\section{Abstract}

Simulation is an inexpensive and fast predictive tool for learning about various processes in advance. In the present work, it has been applied to the deoxygenation and reoxygenation processes in water bodies, due to the presence of organic load. The carbonaceous and nitrogenous demand of water bodies has been modeled, both for stationary and non-stationary regime, considering the advection, diffusion and reaction model. For the non-stationary case, two scenarios are taken into account: one of instantaneous entry (pulse type) of the pollutant and the other, of permanent entry. The solubility of oxygen in water is obtained by equations that consider the influence of temperature, salt content and pressure. The respective programs were developed,

(C) Los autores. Este artículo es publicado por la Revista Campus de la Facultad de Ingeniería y Arquitectura de la Universidad de San Martín de Porres. Este artículo se distribuye en los términos de la Licencia Creative Commons Atribución No-comercial - Compartir-Igual 4.0 Internacional (https://creativecommons.org/licenses/ CC-BY), que permite el uso no comercial, distribución y reproducción en cualquier medio siempre que la obra original sea debidamente citada. Para uso comercial contactar a: revistacampus@usmp.pe. 
calibrated and executed, considering that the organic matter decay curves, expressed in terms of the variation of BOD and oxygen deficit, have a Gaussian-type temporal and spatial evolution. As a result, the curves that describe the instantaneous entry of the pollutant downstream of the discharge point were obtained, turning out to be Gaussian type and, in the case of permanent entry of the pollutant, hyperbolic curves. The proposed models are used to estimate the displacement and concentration levels of the organic load; likewise, the critical oxygen deficit of the water body, important parameters for evaluating water quality.

Key words: modeling, simulation, body of water, advection, diffusion, reaction

\section{Introducción}

Los cuerpos de agua están permanentemente sometidos al vertido de diversos contaminantes; los de carácter inorgánico: sales, ácidos, metales, álcalis, relaves mineros, entre otros; los orgánicos: residuos municipales, residuos industriales, insecticidas, entre los que se encuentra, la materia orgánica proveniente de los procesos industriales. La materia orgánica provoca la disminución del oxígeno disuelto en el agua, afectando negativamente la vida acuática, especialmente, de organismos muy sensibles. Para predecir estos cambios de forma rápida y económica, existen algunas herramientas como el modelamiento y simulación, los cuales se basan en los principios de la conservación de materia que requieren el uso de parámetros experimentales. La importancia del presente trabajo radica en el hecho de ser una herramienta que permite realizar predicciones de la tasa de déficit de oxígeno como consecuencia de la presencia de materia orgánica en los cuerpos de agua. Este procedimiento, actualmente es relevante pues muchos estudios de impacto ambiental exigen que se realicen simulaciones de los posibles impactos generados por el inicio de una actividad económica (Anguiano, 2012 \& Boluda, 2011). A la vez, la simulación se constituye en una herramienta fundamental como punto de partida para la realización de un trabajo experimental, pues el programa a través de los datos reportados, permite orientar el rumbo de la investigación experimental (Benítez, 2013).

Respecto al modelamiento de los procesos de dispersión de contaminantes, se ha desarrollado una serie de investigaciones. González-López y Ramírez-León (2011) implementaron un modelo numérico para simular la hidrodinámica y el transporte de contaminantes en sistemas donde existe vegetación, tanto sumergida como emergente. El objetivo fue reproducir las funciones de filtrado de contaminación y reaereación que cumplen las plantas en cuerpos de agua, como los humedales. Los resultados de campo, en las velocidades de flujo se aprecian un cambio de comportamiento por la restricción al flujo que impone la vegetación. Las concentraciones de demanda bioquímica de oxígeno (DBO) y oxígeno disuelto (OD) varían debido al tiempo de residencia y a la reaereación producida por el intercambio atmosférico y la respiración de las plantas. Concluyeron que el modelo representa de manera óptima el comportamiento del transporte de sustancias disueltas en flujos con presencia de vegetación y que se puede aplicar a la gran variedad de ecosistemas, siendo capaz de predecir la ruta y destino de la contaminación. 
En 2015, Cajas realizó una modelación de los parámetros más importantes de calidad de agua: DBO, coliformes y OD, utilizando el Excel. Para el estudio estableció una red de monitoreo de trece puntos a lo largo de los tres ríos, que comprenden tres sitios de monitoreo continuo y diez sitios de contaminación puntual. Se realizaron dos muestreos en diferentes épocas del año (lluvia y sequía). Indican que el modelo matemático desarrollado se basa en la ecuación de Streeter \& Phelps, la misma que ha permitido obtener el transporte de contaminantes puntuales a sitios de monitoreo continuo; ha sido ya calibrado y verificado en estudios anteriores de manera que se pueda obtener valores confiables. Finalmente, obtuvieron valores de contaminación difusa de las principales variables de calidad de agua en el río Tomebamba antes y después de la zona urbana de la ciudad de Cuenca.

Regalado (2008), desarrolló un modelo matemático riguroso a través de la aplicación de una teoría establecida donde se toma a la calidad de agua superficial como un caso de estudio. Con este parámetro pretendía demostrar cómo los modelos se pueden complicar dependiendo de la rigurosidad y exactitud que se quiera tener al representar el fenómeno. También, presentan soluciones analíticas de algunos de los modelos y propusieron la resolución por el método numérico de diferencias finitas los modelos que no contienen solución analítica, dejando las ecuaciones discretizadas.

Brenner, Shacham y Cutlip, (2005), y Guzmán (2014) presentaron el modelamiento y simulación del proceso de desoxigenación y reoxigenación de los cuerpos de agua a condiciones diversas de temperatura. En la "Guía para la determinación de la zona de mezcla y la evaluación del impacto del vertimiento de aguas residuales tratadas a un cuerpo natural de agua", presentada por la Autoridad Nacional del Agua (ANA, 2017), se describe la metodología de cálculo para determinar la extensión de la zona de mezcla, y las concentraciones de los diferentes parámetros que un vertimiento aporta a un cuerpo natural de agua después de la mezcla, diferenciando los principales tipos de cuerpos receptores: cuerpos de agua lóticos (parte II), lénticos (parte III) y marino costeros (parte IV).

En el presente trabajo se ha realizado el modelamiento y simulación del proceso de desoxigenación y reoxigenación considerando los fenómenos de advección, difusión y reacción, tanto en régimen estacionarios, como no estacionario, con ingreso instantáneo y con ingreso permanente de contaminantes, para lo cual fue necesario el conocimiento de los parámetros de los modelos, que a su vez son funciones de las características hidrodinámicas del río materia de estudio, tal como lo indica Cárdenas (2016).

\section{Método}

Se utilizaron las ecuaciones de conservación de materia en régimen no estacionario y estacionario, los cuales son modificaciones del modelo de StreeterPhelps; los parámetros fueron obtenidos a partir de correlaciones empíricas y mediante el método de momentos estadísticos para el cálculo del coeficiente de dispersión longitudinal. La resolución de los modelos se hizo con el software Polymath. 


\section{Cálculo de parámetros}

\section{Solubilidad del oxígeno}

Según Chapra, (1997) y Zhen-Gang,
(2008), basado en la APHA (American Public Health Association), la cual es empleada por los modelos QUALK2K la concentración de oxígeno en el agua pura, se calcula mediante la ecuación:

$$
\ln O_{S f}=-139.3441+\frac{1.575701 \times 10^{5}}{T_{K}}-\frac{6.642308 \times 10^{7}}{T_{K}^{2}}+\frac{1.2438 \times 10^{10}}{T_{K}^{3}}-\frac{8.621949 \times 10^{11}}{T_{K}^{4}}
$$

y en presencia de sal se utiliza la ecuación:

$$
\ln O_{S S}=L n O_{s f}-S\left(1.7674 \times 10^{-2}-\frac{1.0754 \times 10^{1}}{T_{K}}+\frac{2.1407 \times 10^{3}}{T_{K}^{4}}\right)
$$

La dependencia de la solubilidad del oxígeno con la presión según (Rueda Valdivia, s. f.) está dada por:

$$
O_{s p}=O_{s f} \times P\left[\frac{\left(1-\frac{P_{w v}}{P}\right)(1-\theta \times P)}{\left(1-P_{w v}\right)(1-\theta)}\right]
$$

La presión de vapor de agua se obtiene mediante:

$\ln P_{w v}=11.8571-\frac{3840.70}{T_{K}}-\frac{216.961}{T_{K}^{2}}$

El factor de corrección de la temperatura es

$\theta=0.0000975-1.426 \times 10^{-5} \times T+6.436 \times 10^{-8} \times T^{2}$

\section{Coeficiente de dispersión}

Según Potter (2002), el coeficiente de dispersión longitudinal se calcula mediante la derivada de la varianza espacial respecto al tiempo, es decir:

$$
D_{X}=\frac{1}{2} \frac{d \sigma_{(x)}^{2}}{d t}
$$

Desde el punto de vista experimental, es mucho más factible encontrar la varianza temporal, en lugar de la varianza espacial, cuya relación está dada por la ecuación:

$$
\sigma_{(x)}^{2}=U^{2} \times \sigma_{(t)}^{2}
$$

La varianza temporal, la cual es la medida de la dispersión alrededor del centroide de la concentración promediada a través de la sección transversal, se define como:

$$
\sigma_{t}^{2}=\frac{\int_{-\infty}^{\infty}(t-\bar{t})^{2} C(x, t) d t}{\int_{-\infty}^{\infty} C(x, t) d t}
$$

Donde el tiempo de travesía del centroide t está dado por:

$$
\bar{t}=\frac{\int_{-\infty}^{\infty} t \times C(x, t) d t}{\int_{-\infty}^{\infty} C(x, t) d t}
$$

El denominador de las ecuaciones (8) y (9) se denomina momento estadístico de orden cero. La ecuación (9) se denomina momento estadístico de primer orden. La ecuación (8), se denomina momento estadístico de segundo orden. Por tanto, el coeficiente de dispersión se obtiene en forma explícita, mediante:

$$
D_{x}=\frac{1}{2} U^{2} \frac{\sigma_{t}^{2}\left(x_{2}\right)-\sigma_{t}^{2}\left(x_{1}\right)}{t_{2}-t_{1}}
$$

Donde $\sigma_{(t)}^{2}$ es la varianza temporal de la concentración del trazador a través de una sección transversal en los lugares especificados y a lo largo del río.

Para determinar el coeficiente de dispersión longitudinal se requiere hacer uso de las ecuaciones (6) al (10)

El momento estadístico de orden cero se calcula mediante: 


$$
\mu_{0}=\sum_{1}^{n}\left[\frac{C\left(t_{i}\right)+C\left(t_{i-1}\right)}{2}\right] \times \Delta t_{i}
$$

El momento estadístico de orden uno se obtiene mediante:

$$
\mu_{1}=t_{p}=\frac{\sum_{1}^{n} t_{i}\left[\frac{C\left(t_{i}\right)+C\left(t_{i-1}\right)}{2}\right]}{\mu_{0}} \times \Delta t_{i}
$$

\section{Parámetros cinéticos}

Los parámetros cinéticos de los procesos de desoxigenación y reoxigenación han sido tomados de la literatura especializada. Se plantea las ecuaciones de los modelos propuestos en base a las ecuaciones de

$$
k_{a}=3.93 \times \frac{U^{0.5}}{H^{1.5}} \quad 0.305 \leq H \leq 9.14
$$

Churchill et. al. (1962), basado en las tasas de reaireación observadas aguas debajo de presas, en las cuales se conocía el déficit de oxígeno. No es aplicable a

$$
k_{a}=3.93 \times \frac{U^{0.969}}{H^{1.673}} \quad 0.61 \leq H \leq 3.35
$$

La tasa de desoxigenación por reacción química presenta Amarilla (2017), con valores específicos obtenido en trabajo de campo cuyos valores fluctúan desde 0.08 a $4.24 \mathrm{~d}^{-1}$.

\section{Modelo matemático}

Modelos con dispersión-advecciónreacción en régimen no estacionario con
El momento estadístico de orden dos se obtiene mediante:

$$
\mu_{2}=\sigma_{t}^{2}=\frac{\sum_{1}^{n}\left(t_{i}-t_{p}\right)^{2}\left[\frac{C\left(t_{i}\right)+C\left(t_{i-1}\right)}{2}\right]}{\mu_{0}} \times \Delta t_{i}
$$

conservación de materia. Orduz (2016) presenta una serie de correlaciones cinéticas del proceso de reoxigenación de los flujos de agua.

O'Connor y Dobbins (1958) indican que dicha constante está dada por:

$$
0.15 \leq U \leq 0.49 \quad 0.05 \leq k_{a} \leq 12.22
$$

ríos con pequeñas cascadas (fenómeno de burbujas). En la referencia se presentan muchas otras correlaciones.

$$
0.55 \leq U \leq 1.5 \quad 0.225 \leq k_{a} \leq 5.56
$$

cargas puntuales con presencia de carga orgánica conteniendo DBOC y DBON (Pérez, 2017; Zoutien, 2012; Zúñiga, 2014).

$$
\frac{\partial L_{C}}{\partial t}=E \frac{\partial^{2} L_{C}}{\partial x^{2}}-U \frac{\partial L_{C}}{\partial x}-k_{r} L_{C}+W_{C}^{1}
$$

$$
\frac{\partial L_{N}}{\partial t}=E \frac{\partial^{2} L_{N}}{\partial x^{2}}-U \frac{\partial L_{N}}{\partial x}-k_{r N} L_{N}+W_{N}^{1}
$$

Con las condiciones iniciales para ingreso de contaminante instantáneo

$$
\begin{array}{llllll}
t=0 & L_{i}=0 \quad D_{i}=D_{I N} \forall x & x=0 & L_{0} \neq 0 & D_{0}=D_{I N} \forall t \\
x=x_{1} & L_{1}=0 & D_{1}=D_{I N} \forall t & x=x_{n} & \frac{\partial L}{\partial r}=0 & \frac{\partial D}{\partial r}=0 \quad \forall t
\end{array}
$$


Este sistema de ecuaciones diferenciales transformación a un sistema de ecuaciones parciales se resuelve por el proceso de diferenciales ordinarias.

$$
\begin{gathered}
\frac{d L_{C i}}{d t}=E\left(\frac{L_{C i+1}-2 L_{C i}+L_{C i-1}}{\Delta x^{2}}\right)-U \frac{\left(L_{C i}-L_{C i-1}\right)}{\Delta x}-k_{r} L_{C i}+W_{C i}^{1} \\
\frac{d L_{N i}}{d t}=E\left(\frac{L_{N i+1}-2 L_{N i}+L_{N i-1}}{\Delta x^{2}}\right)-U \frac{\left(L_{N i}-L_{N i-1}\right)}{\Delta x}-k_{r n} L_{N i}+W_{N i}^{1} \\
\frac{d D_{i}}{d t}=E\left(\frac{D_{i+1}-2 D_{i}+D_{i-1}}{\Delta x^{2}}\right)-U \frac{\left(D_{i}-D_{i-1}\right)}{\Delta x}-k_{a} D_{i}+k_{d} L_{C i}+k_{n} L_{N i}+\sum W_{2 i}
\end{gathered}
$$

Modelos con dispersión-advección- cargas puntuales con presencia de carga reacción en régimen estacionario con orgánica conteniendo DBOC y DBON.

$$
\begin{gathered}
E \frac{d^{2} L_{C}}{d x^{2}}-U \frac{d L_{C}}{d x}-k_{r} L_{C}+W_{L}{ }^{1}=0 \\
E \frac{d^{2} L_{N}}{d x^{2}}-U \frac{d L_{N}}{d x}-k_{r N} L_{N}+W_{N}^{1}=0 \\
E \frac{d^{2} D}{d x^{2}}-U \frac{d D}{d x}-k_{a} D+k_{d} L_{C}+k_{n} L_{N}+\sum W_{2}=0
\end{gathered}
$$

Con las condiciones de frontera:

$$
x=0 \quad L_{C 0}=0 \quad L_{N 0}=0 \quad D_{0}=D_{I N} \quad x=x_{1} \quad \frac{\partial L_{C}}{\partial x}=0 \quad \frac{\partial L_{N 0}}{\partial x}=0 \quad D_{0}=D_{I N}
$$

Este sistema de ecuaciones diferenciales permite transformar a un sistema de se resuelve por desratización, hecho que ecuaciones algebraicas lineales.

$$
\begin{aligned}
& E\left(\frac{L_{C i+1}-2 L_{C i}+L_{C i-1}}{\Delta x^{2}}\right)-U \frac{\left(L_{C i}-L_{C i-1}\right)}{\Delta x}-k_{r} L_{C i}+W_{C i}^{1}=0 \\
& E\left(\frac{L_{N i+1}-2 L_{N i}+L_{N i-1}}{\Delta x^{2}}\right)-U \frac{\left(L_{N i}-L_{N i-1}\right)}{\Delta x}-k_{r n} L_{N i}+W_{N i}^{1}=0 \\
& E\left(\frac{D_{i+1}-2 D_{i}+D_{i-1}}{\Delta x^{2}}\right)-U \frac{\left(D_{i}-D_{i-1}\right)}{\Delta x}-k_{a} D_{i}+k_{d} L_{C i}+k_{n} L_{N i}+\sum W_{2 i}=0
\end{aligned}
$$

Este sistema de ecuaciones puede parametrizada. ser modificado ligeramente para ser

$$
\begin{gathered}
L_{C i+1}-2 L_{C i}+L_{C i-1}-\frac{U \times \Delta x}{E}\left(L_{C i}-L_{C i-1}\right)-\frac{\Delta x^{2}}{E} k_{r} L_{C i}+\frac{\Delta x^{2}}{E} W_{C i}^{1}=0 \\
L_{N i+1}-2 L_{N i}+L_{N i-1}-\frac{U \times \Delta x}{E}\left(L_{N i}-L_{N i-1}\right)-\frac{\Delta x^{2}}{E} k_{r n} L_{N i}+\frac{\Delta x^{2}}{E} W_{N i}^{1}=0 \\
D_{i+1}-2 D_{i}+D_{i-1}-\frac{U \times \Delta x}{E}\left(D_{i}-D_{i-1}\right)-\frac{\Delta x^{2}}{E} k_{a} D_{i}+\frac{\Delta x^{2}}{E} k_{d} L_{C i}+\frac{\Delta x^{2}}{E} k_{n} L_{N i}+\frac{\Delta x^{2}}{E} \sum W_{2 i}=0
\end{gathered}
$$


Las ecuaciones anteriores se pueden simplificar del siguiente modo:

$$
\begin{gathered}
L_{C i+1}+\left(-2-\frac{U \times \Delta x}{E}-\frac{\Delta x^{2}}{E} k_{r}\right) L_{C i}+\frac{U \times \Delta x}{E} L_{C i-1}+\frac{\Delta x^{2}}{E} W_{C i}^{1}=0 \\
L_{N i+1}+\left(-2-\frac{U \times \Delta x}{E}-\frac{\Delta x^{2}}{E} k_{r n}\right) L_{N i}+\frac{U \times \Delta x}{E} L_{N i-1}+\frac{\Delta x^{2}}{E} W_{N i}^{1}=0 \\
D_{i+1}+\left(-2-\frac{U \times \Delta x}{E}-\frac{\Delta x^{2}}{E} k_{a}\right) D_{i}+\frac{U \times \Delta x}{E} D_{i-1}+\frac{\Delta x^{2}}{E} k_{d} L_{C i}+\frac{\Delta x^{2}}{E} k_{n} L_{N i}+\frac{\Delta x^{2}}{E} \sum W_{2 i}=0
\end{gathered}
$$

Parametrizando el sistema anterior

$$
\begin{aligned}
& A_{1}=-2-\frac{U \times \Delta x}{E}-\frac{\Delta x^{2}}{E} k_{r} \quad B=\frac{U \times \Delta x}{E} \quad F_{i}=\frac{\Delta x^{2}}{E} W_{C i}^{1} \\
& A_{2}=-2-\frac{U \times \Delta x}{E}-\frac{\Delta x^{2}}{E} k_{r n} \quad G_{i}=\frac{\Delta x^{2}}{E} W_{N i}^{1} \\
& A_{3}=-2-\frac{U \times \Delta x}{E}-\frac{\Delta x^{2}}{E} k_{a} \quad H=\frac{\Delta x^{2}}{E} k_{d} \quad M=\frac{\Delta x^{2}}{E} k_{n} \quad P_{i}=\frac{\Delta x^{2}}{E} \sum W_{2 i}
\end{aligned}
$$

Las ecuaciones (29), (30) y (31) se transforman en:

$$
\begin{aligned}
& L_{C i+1}+A_{1} L_{C i}+B L_{C i-1}+F_{i}=0 \\
& L_{N i+1}+A_{2} L_{N i}+B L_{N i-1}+G_{i}=0 \\
& D_{i+1}+A_{3} D_{i}+B D_{i-1}+H L_{C i}+M L_{N i}+P_{i}=0
\end{aligned}
$$

Las ecuaciones (32), (33) y (34) constituyen un sistema de ecuaciones algebraicas lineales, los cuales se resuelven con la ayuda del software polymath.

\section{Resultados}

Como resultado de la ejecución de los programas se obtiene las tablas y gráficos que se muestran a continuación: Proceso en régimen no estacionario: Se ha desarrollado el programa p_reg_no_estac. pol y se ejecuta el programa considerando ingreso instantáneo e ingreso permanente. 
Tabla 1

Resultados de la ejecución del programa que muestra las variables principales de entrada y salida y sus valores iniciales y finales.

\begin{tabular}{|c|c|c|c|}
\hline Denominación & Variable & Valor inicial & Valor final \\
\hline$\overline{\text { Tiempo }}$ & $\mathrm{T}$ & 0 & 40 \\
\hline \multirow[t]{10}{*}{ DBO carbonácea } & L1 & 80 & 0.0874754 \\
\hline & $\mathrm{L} 2$ & 0 & 0.2867201 \\
\hline & L3 & 0 & 0.5137871 \\
\hline & $\mathrm{L} 4$ & 0 & 0.6942439 \\
\hline & $\mathrm{L} 5$ & 0 & 0.7121119 \\
\hline & L6 & 0 & 0.5617951 \\
\hline & L7 & 0 & 0.3903157 \\
\hline & L8 & 0 & 0.2381132 \\
\hline & L9 & 0 & 0.131709 \\
\hline & L10 & 0 & 0.0670808 \\
\hline \multirow{10}{*}{ DBO nitrogenada } & N1 & 20 & 0.1023794 \\
\hline & N2 & 0 & 0.3218527 \\
\hline & N3 & 0 & 0.6219303 \\
\hline & N4 & 0 & 0.8647035 \\
\hline & N5 & 0 & 0.8900363 \\
\hline & N6 & 0 & 0.7140409 \\
\hline & N7 & 0 & 0.5037744 \\
\hline & N8 & 0 & 0.3367482 \\
\hline & N9 & 0 & 0.1969921 \\
\hline & N10 & 0 & 0.1091544 \\
\hline \multirow{11}{*}{ Déficit de oxígeno } & D1 & 0.2 & 0.1174671 \\
\hline & D2 & 0.2 & 0.2347159 \\
\hline & D3 & 0.2 & 0.4158436 \\
\hline & D4 & 0.2 & 0.550601 \\
\hline & D5 & 0.2 & 0.5465944 \\
\hline & D6 & 0.2 & 0.4490619 \\
\hline & D7 & 0.2 & 0.3062444 \\
\hline & D8 & 0.2 & 0.1886351 \\
\hline & D9 & 0.2 & 0.1117885 \\
\hline & D10 & 0.2 & 0.0575601 \\
\hline & D11 & 0.2 & 0.0575601 \\
\hline Temperatura & $\mathrm{T}$ & 20 & 20 \\
\hline Presión & $\mathrm{P}$ & 0.7210526 & 0.7210526 \\
\hline Temperatura absoluta & TK & 293.15 & 293.15 \\
\hline Presión de vapor & PWV & 0.2873919 & 0.2873919 \\
\hline Concentración de sal & $\mathrm{S}$ & 25 & 25 \\
\hline Parámetro & THETA & 0.0007155 & 0.0007155 \\
\hline Concentración de oxígeno en agua salada & CS1 & 9.092426 & 9.092426 \\
\hline Concentración de oxígeno a la presión $\mathrm{P}$ & CS2 & 7.845544 & 7.845544 \\
\hline Velocidad del flujo de agua & $\mathrm{U}$ & 2 & 2 \\
\hline Demanda carbonácea aguas arriba & L0 & 0 & 0 \\
\hline Demanda nitrogenada aguas arriba & No & 0 & 0 \\
\hline Déficit inicial & D0 & 0.2 & 0.2 \\
\hline Constante de reaireación & ka & 0.2 & 0.2 \\
\hline Constante de remoción de DBOC por reacción & $\mathrm{kd}$ & 0.06 & 0.06 \\
\hline Constante de remoción de DBON por reacción & $\mathrm{kn}$ & 0.04 & 0.04 \\
\hline Tasa de remoción de materia carbonácea & $\mathrm{kr}$ & 0.08 & 0.08 \\
\hline Tasa de remoción de materia nitrogenada & krn & 0.04 & 0.04 \\
\hline
\end{tabular}




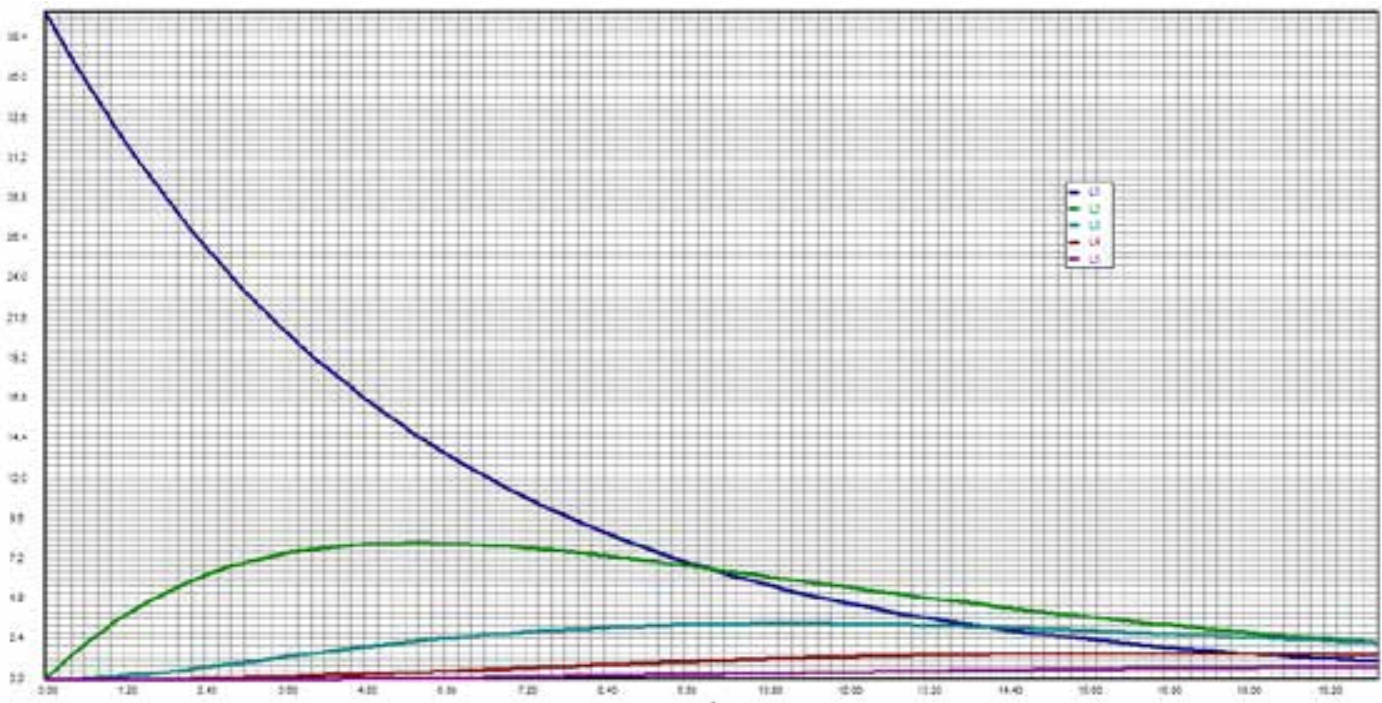

Figura 1. Evolución de la demanda bioquímica de oxígeno carbonácea en función al tiempo.

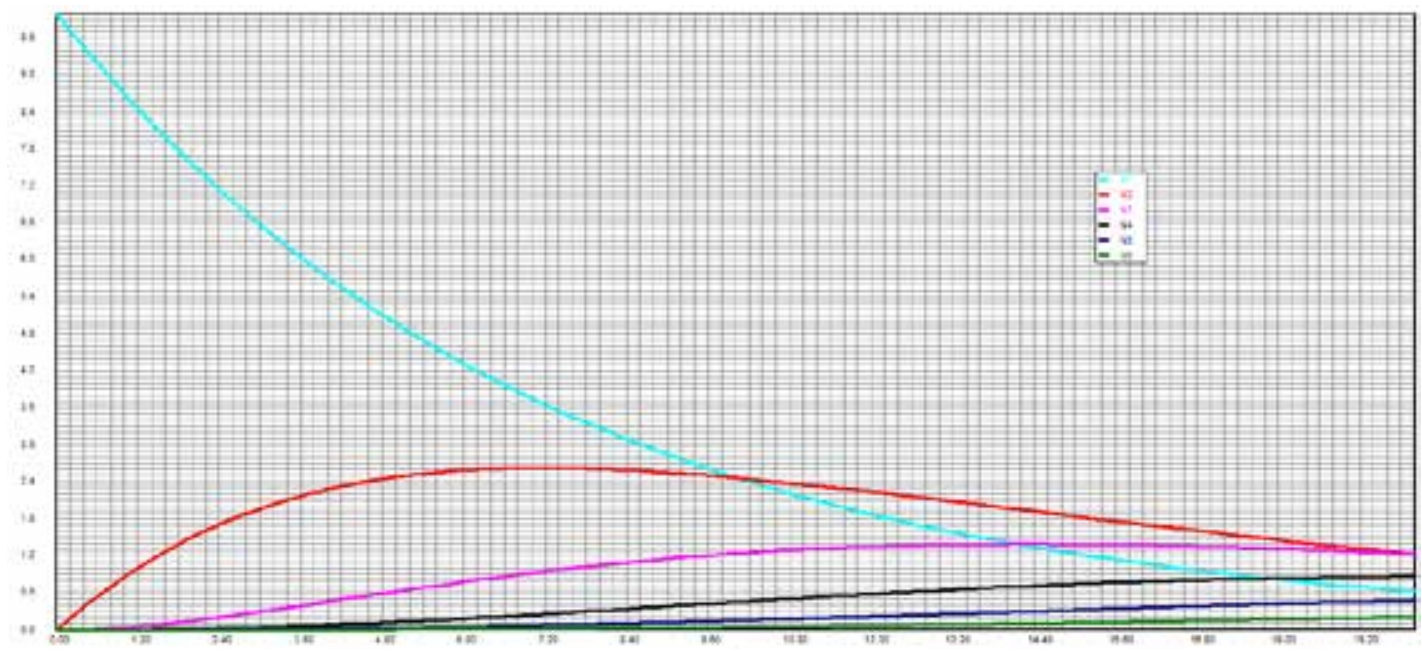

Figura 2. Evolución de la demanda bioquímica de oxígeno nitrogenada en función al tiempo.

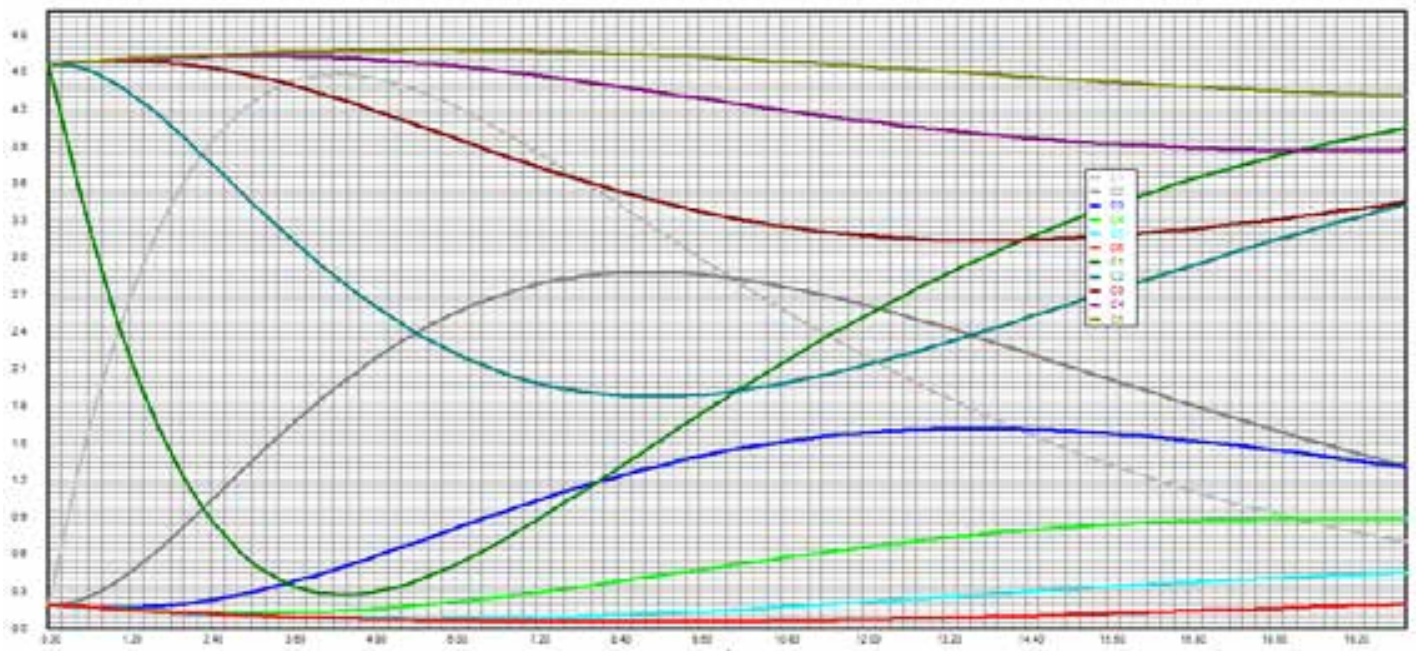

Figura 3.Evolución del déficit y concentración de oxígeno en función al tiempo para cada posición longitudinal. 
De la Tabla 1 y de las Figuras 1, 2 y 3 se observa que en régimen no estacionario, la concentración de la demanda bioquímica de oxígeno carbonácea y nitrogenada y el déficit de oxígeno expresados en ppm, considerando un ingreso tipo pulso se obtiene las curvas de descenso logarítmico para la demanda carbonácea y nitrogenada y una curva creciente-decreciente referido al déficit de oxígeno. Este hecho se explica del siguiente modo: una vez que ingresa la materia orgánica, comienza el proceso de oxidación de la materia orgánica, consecuentemente, disminuye su concentración; en el caso del déficit, el oxígeno disuelto es consumido por el ingreso de la carga orgánica; llegando a un valor crítico, hasta el punto de consumirse totalmente; sin embargo, conforme pasa el tiempo y la distancia, el déficit comienza a disminuir, lo cual significa que la concentración de oxígeno se va restaurando paulatinamente, hasta recuperar sus cualidades originales en el cuerpo de agua.

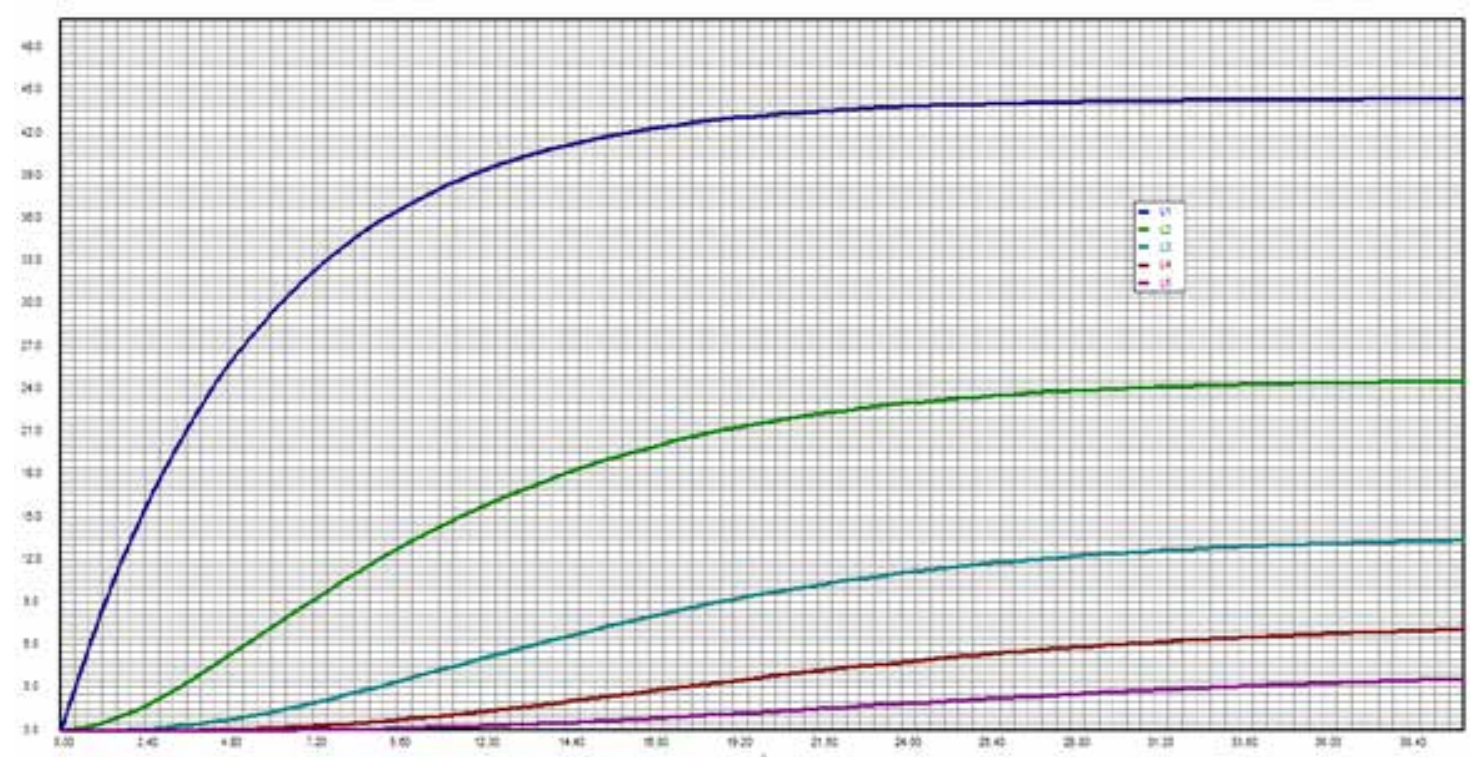

Figura 4. Evolución de la carga orgánica carbonácea función al tiempo para cada posición longitudinal, con ingreso permanente aguas abajo.

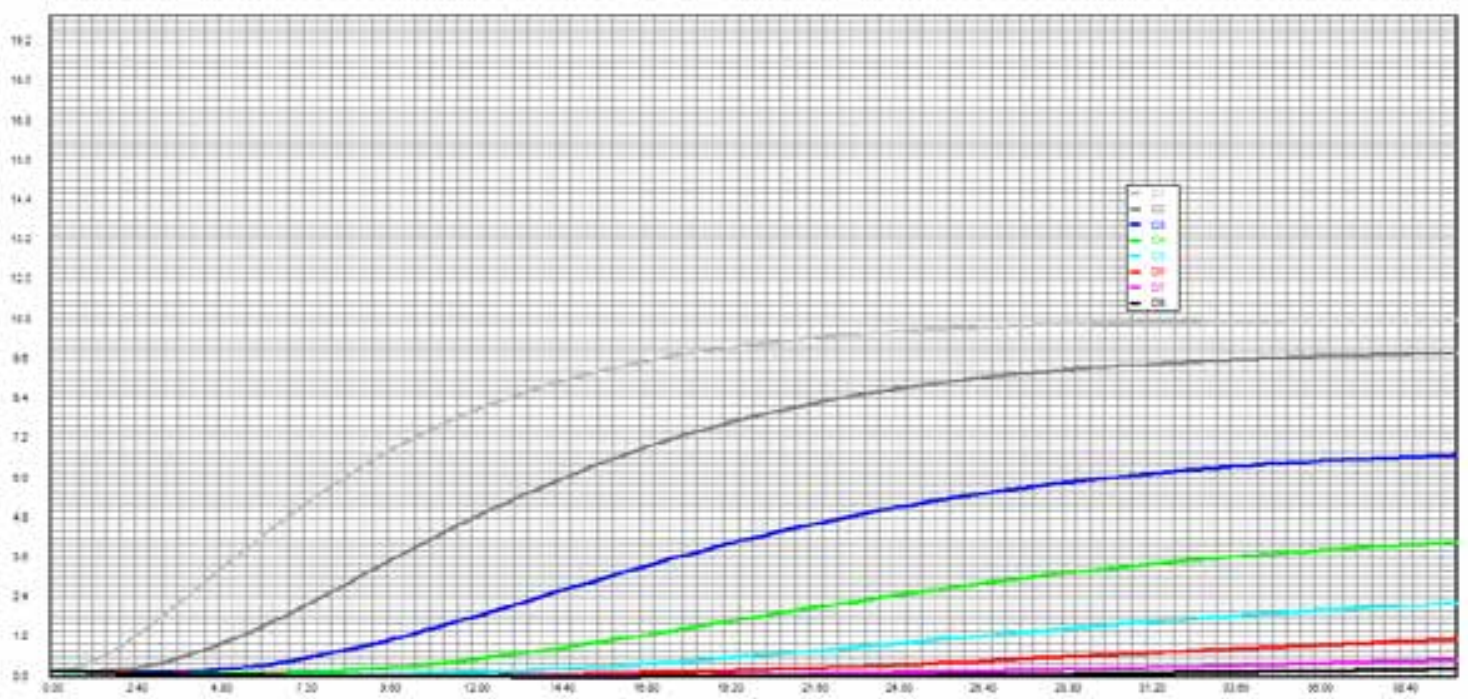

Figura 5. Evolución del déficit de oxígeno función al tiempo para cada posición longitudinal, con ingreso permanente aguas abajo. 
En las Figuras 4 y 5 se simula el caso en el cual en un determinado punto hay un ingreso permanente de contaminante al cuerpo de agua principal. La concentración de la DBO y el déficit de oxígeno, se estabilizan en ciertos valores fijos, característica de los procesos discontinuos. Estos valores dependen de factores como la hidrodinámica, de las condiciones del flujo principal, así como de las características del efluente; la fiabilidad de los parámetros utilizados juega un papel importante en los resultados obtenidos. Proceso en régimen estacionario: Se ha desarrollado el programa p_reg_estac.pol y se ejecuta el programa considerando ingreso instantáneo e ingreso permanente.

Tabla 2

Resultados de la ejecución del programa p_reg_estac.pol que muestra las variables principales de entrada $y$ salida $y$ sus valores iniciales $y$ finales.

\begin{tabular}{lrr}
\hline & Variable & \multicolumn{1}{c}{ Valor } \\
\hline Demanda bioquímica carbonácea & L1 & 56.95114 \\
& L2 & 40.55157 \\
L3 & 28.86636 \\
Demanda bioquímica nitrogenada & L4 & 20.5682 \\
& L5 & 14.66618 \\
& L20 & 0.1224003 \\
Déficit de oxigeno & $\mathrm{N} 1$ & 16.61854 \\
& $\mathrm{~N} 2$ & 13.79917 \\
& $\mathrm{~N} 3$ & 11.46305 \\
& $\mathrm{~N} 4$ & 9.548733 \\
& $\mathrm{~N} 20$ & 0.5643165 \\
& $\mathrm{D} 1$ & 10.28356 \\
$\mathrm{D} 2$ & 12.58259 \\
& $\mathrm{D} 3$ & 11.74536 \\
& $\mathrm{D} 4$ & 9.894044 \\
& $\mathrm{D} 20$ & 0.1978248 \\
\hline
\end{tabular}

Nota. Elaborado a partir de la ejecución del programa

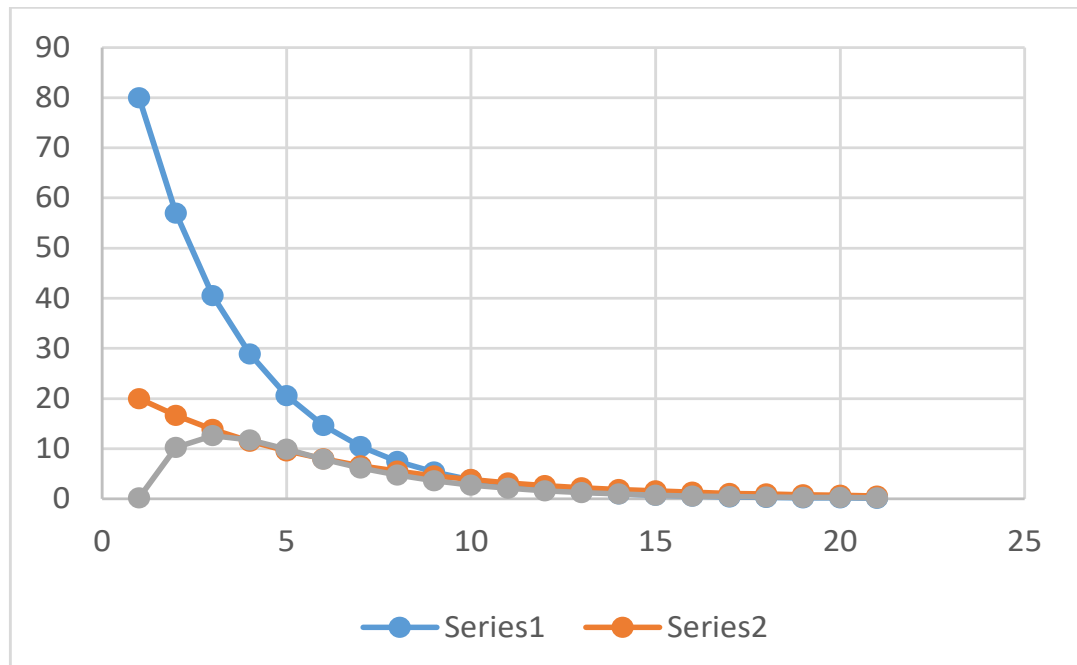

Figura 6. Concentracion de carga orgánica carbonácea, nitrogenada y déficit de oxígeno como función de la posición espacial. 
En la Tabla 2 y Figura 6 se observan los resultados de la ejecución del programa constituido por un sistema de ecuaciones algebraicas lineales. La curva de descenso de la DBO carbonosa y DBO nitrogenada tienen un descenso logarítmico, dado que la evidencia empírica muestra quelas reacciones de oxidación que se producen en el cuerpo de agua, son de primer orden. Respecto al déficit de oxígeno, tiene la característica curva creciente decreciente, alcanzando un valor crítico a una determinada distancia.

\section{Conclusiones}

Se han desarrollados los modelos de advección-difusión-reacción para un régimen no estacionario y estacionario de un cuerpo de agua, considerando el ingreso instantáneo y permanente de contaminantes. Se ha considerado, el aporte de cargas puntuales y de generación de materia orgánica carbonácea y nitrogenada y/o consumo de oxígeno. Los conocimientos de los parámetros fisicoquímicos e hidrodinámicos han permitido predecir la evolución espacial y/o temporal de la concentración de la materia orgánica y el déficit de oxígeno. Los dos modelos desarrollados se encuentran calibrados y pueden ser usados para predecir la contaminación por materia orgánica en situaciones particulares. 


\section{ANEXOS}

\section{Anexo 1: Programa P_REG_NO_ESTAC.pol}

\# Balance de materia de carga orgánica carbonácea

$\mathrm{d}(\mathrm{L} 1) / \mathrm{d}(\mathrm{t})=-\mathrm{U}^{*}(\mathrm{~L} 1-\mathrm{L} 0) / \mathrm{DX}+\mathrm{E}^{*}\left(\mathrm{~L} 2-2^{*} \mathrm{~L} 1+\mathrm{L} 0\right) / \mathrm{DX}^{\wedge} 2-\mathrm{kr}^{*} \mathrm{~L} 1+\mathrm{WC} 1$

$\mathrm{d}(\mathrm{L} 2) / \mathrm{d}(\mathrm{t})=-\mathrm{U}^{*}(\mathrm{~L} 2-\mathrm{L} 1) / \mathrm{DX}+\mathrm{E}^{*}\left(\mathrm{~L} 3-2^{*} \mathrm{~L} 2+\mathrm{L} 1\right) / \mathrm{DX}^{\wedge} 2-\mathrm{kr}^{*} \mathrm{~L} 2+\mathrm{WC} 2$

$\mathrm{d}(\mathrm{L} 3) / \mathrm{d}(\mathrm{t})=-\mathrm{U}^{*}(\mathrm{~L} 3-\mathrm{L} 2) / \mathrm{DX}+\mathrm{E}^{*}\left(\mathrm{~L} 4-2^{*} \mathrm{~L} 3+\mathrm{L} 2\right) / \mathrm{DX} \wedge 2-\mathrm{kr}^{*} \mathrm{~L} 3+\mathrm{WC} 3$

$\mathrm{d}(\mathrm{L} 4) / \mathrm{d}(\mathrm{t})=-\mathrm{U}^{*}(\mathrm{~L} 4-\mathrm{L} 3) / \mathrm{DX}+\mathrm{E}^{*}\left(\mathrm{~L} 5-2^{*} \mathrm{~L} 4+\mathrm{L} 3\right) / \mathrm{DX}^{\wedge} 2-\mathrm{kr}^{*} \mathrm{~L} 4+\mathrm{WC} 4$

$\mathrm{d}(\mathrm{L} 5) / \mathrm{d}(\mathrm{t})=-\mathrm{U}^{*}(\mathrm{~L} 5-\mathrm{L} 4) / \mathrm{DX}+\mathrm{E}^{*}(\mathrm{~L} 6-2 * \mathrm{~L} 5+\mathrm{L} 4) / \mathrm{DX} \wedge 2-\mathrm{kr}^{*} \mathrm{~L} 5+\mathrm{WC} 5$

$\mathrm{d}(\mathrm{L} 6) / \mathrm{d}(\mathrm{t})=-\mathrm{U}^{*}(\mathrm{~L} 6-\mathrm{L} 5) / \mathrm{DX}+\mathrm{E}^{*}\left(\mathrm{~L} 7-2^{*} \mathrm{~L} 6+\mathrm{L} 5\right) / \mathrm{DX}^{\wedge} 2-\mathrm{kr}^{*} \mathrm{~L} 6+\mathrm{WC} 6$

$\mathrm{d}(\mathrm{L} 7) / \mathrm{d}(\mathrm{t})=-\mathrm{U}^{*}(\mathrm{~L} 7-\mathrm{L} 6) / \mathrm{DX}+\mathrm{E}^{*}\left(\mathrm{~L} 8-2^{*} \mathrm{~L} 7+\mathrm{L} 6\right) / \mathrm{DX}^{\wedge} 2-\mathrm{kr}^{*} \mathrm{~L} 7+\mathrm{WC} 7$

$\mathrm{d}(\mathrm{L} 8) / \mathrm{d}(\mathrm{t})=-\mathrm{U}^{*}(\mathrm{~L} 8-\mathrm{L} 7) / \mathrm{DX}+\mathrm{E}^{*}\left(\mathrm{~L} 9-2^{*} \mathrm{~L} 8+\mathrm{L} 7\right) / \mathrm{DX} \wedge 2-\mathrm{kr}^{*} \mathrm{~L} 8+\mathrm{WC} 8$

$\mathrm{d}(\mathrm{L} 9) / \mathrm{d}(\mathrm{t})=-\mathrm{U}^{*}(\mathrm{~L} 9-\mathrm{L} 8) / \mathrm{DX}+\mathrm{E}^{*}\left(\mathrm{~L} 10-2^{*} \mathrm{~L} 9+\mathrm{L} 8\right) / \mathrm{DX} \wedge 2-\mathrm{kr}^{*} \mathrm{~L} 9+\mathrm{WC} 9$

$\mathrm{d}(\mathrm{L} 10) / \mathrm{d}(\mathrm{t})=-\mathrm{U}^{*}(\mathrm{~L} 10-\mathrm{L} 9) / \mathrm{DX}+\mathrm{E}^{*}\left(\mathrm{~L} 11-2^{*} \mathrm{~L} 10+\mathrm{L} 9\right) / \mathrm{DX}^{\wedge} 2-\mathrm{kr}^{*} \mathrm{~L} 10+\mathrm{WC} 10$

\# Balance de materia de carga orgánica nitrogenada

$\mathrm{d}(\mathrm{N} 1) / \mathrm{d}(\mathrm{t})=-\mathrm{U}^{*}(\mathrm{~N} 1-\mathrm{N} 0) / \mathrm{DX}+\mathrm{E}^{*}\left(\mathrm{~N} 2-2^{*} \mathrm{~N} 1+\mathrm{N} 0\right) / \mathrm{DX}^{\wedge} 2-\mathrm{krn}^{*} \mathrm{~N} 1+\mathrm{WN} 1$

$\mathrm{d}(\mathrm{N} 2) / \mathrm{d}(\mathrm{t})=-\mathrm{U}^{*}(\mathrm{~N} 2-\mathrm{N} 1) / \mathrm{DX}+\mathrm{E}^{*}\left(\mathrm{~N} 3-2^{*} \mathrm{~N} 2+\mathrm{N} 1\right) / \mathrm{DX}^{\wedge} 2-\mathrm{krn}^{*} \mathrm{~N} 2+\mathrm{WN} 2$

$\mathrm{d}(\mathrm{N} 3) / \mathrm{d}(\mathrm{t})=-\mathrm{U}^{*}(\mathrm{~N} 3-\mathrm{N} 2) / \mathrm{DX}+\mathrm{E}^{*}\left(\mathrm{~N} 4-2^{*} \mathrm{~N} 3+\mathrm{N} 2\right) / \mathrm{DX} \wedge 2-\mathrm{krn} * \mathrm{~N} 3+\mathrm{WN} 3$

$\mathrm{d}(\mathrm{N} 4) / \mathrm{d}(\mathrm{t})=-\mathrm{U}^{*}(\mathrm{~N} 4-\mathrm{N} 3) / \mathrm{DX}+\mathrm{E}^{*}\left(\mathrm{~N} 5-2^{*} \mathrm{~N} 4+\mathrm{N} 3\right) / \mathrm{DX} \wedge 2-\mathrm{krn}^{*} \mathrm{~N} 4+\mathrm{WN} 4$

$\mathrm{d}(\mathrm{N} 5) / \mathrm{d}(\mathrm{t})=-\mathrm{U}^{*}(\mathrm{~N} 5-\mathrm{N} 4) / \mathrm{DX}+\mathrm{E}^{*}\left(\mathrm{~N} 6-2^{*} \mathrm{~N} 5+\mathrm{N} 4\right) / \mathrm{DX} \wedge 2-\mathrm{krn} * \mathrm{~N} 5+\mathrm{WN} 5$

$\mathrm{d}(\mathrm{N} 6) / \mathrm{d}(\mathrm{t})=-\mathrm{U}^{*}(\mathrm{~N} 6-\mathrm{N} 5) / \mathrm{DX}+\mathrm{E}^{*}\left(\mathrm{~N} 7-2^{*} \mathrm{~N} 6+\mathrm{N} 5\right) / \mathrm{DX} \wedge 2-\mathrm{krn}{ }^{*} \mathrm{~N} 6+\mathrm{WN} 6$

$\mathrm{d}(\mathrm{N} 7) / \mathrm{d}(\mathrm{t})=-\mathrm{U}^{*}(\mathrm{~N} 7-\mathrm{N} 6) / \mathrm{DX}+\mathrm{E}^{*}\left(\mathrm{~N} 8-2^{*} \mathrm{~N} 7+\mathrm{N} 6\right) / \mathrm{DX} \wedge 2-\mathrm{krn}{ }^{*} \mathrm{~N} 7+\mathrm{WN} 7$

$\mathrm{d}(\mathrm{N} 8) / \mathrm{d}(\mathrm{t})=-\mathrm{U}^{*}(\mathrm{~N} 8-\mathrm{N} 7) / \mathrm{DX}+\mathrm{E}^{*}\left(\mathrm{~N} 9-2^{*} \mathrm{~N} 8+\mathrm{N} 7\right) / \mathrm{DX} \wedge 2-\mathrm{krn}^{*} \mathrm{~N} 8+\mathrm{WN} 8$

$\mathrm{d}(\mathrm{N} 9) / \mathrm{d}(\mathrm{t})=-\mathrm{U}^{*}(\mathrm{~N} 9-\mathrm{N} 8) / \mathrm{DX}+\mathrm{E}^{*}(\mathrm{~N} 10-2 * \mathrm{~N} 9+\mathrm{N} 8) / \mathrm{DX} \wedge 2-\mathrm{krn}^{*} \mathrm{~N} 9+\mathrm{WN} 9$

$\mathrm{d}(\mathrm{N} 10) / \mathrm{d}(\mathrm{t})=-\mathrm{U}^{*}(\mathrm{~N} 10-\mathrm{N} 9) / \mathrm{DX}+\mathrm{E}^{*}\left(\mathrm{~L} 11-2^{*} \mathrm{~N} 10+\mathrm{N} 9\right) / \mathrm{DX} \wedge 2-\mathrm{krn} * \mathrm{~N} 10+\mathrm{WN} 10$

\# Balance de materia de déficit de oxígeno

$\mathrm{d}(\mathrm{D} 1) / \mathrm{d}(\mathrm{t})=-\mathrm{U}^{*}(\mathrm{D} 1-\mathrm{D} 0) / \mathrm{DX}+\mathrm{E}^{*}\left(\mathrm{D} 2-2^{*} \mathrm{D} 1+\mathrm{D} 0\right) / \mathrm{DX} \wedge 2-\mathrm{ka}^{*} \mathrm{D} 1+\mathrm{kd} * \mathrm{~L} 1+$ $\mathrm{kn}^{*} \mathrm{~N} 1+\mathrm{SW} 21$

$\mathrm{d}(\mathrm{D} 2) / \mathrm{d}(\mathrm{t})=-\mathrm{U}^{*}(\mathrm{D} 2-\mathrm{D} 1) / \mathrm{DX}+\mathrm{E}^{*}\left(\mathrm{D} 3-2^{*} \mathrm{D} 2+\mathrm{D} 1\right) / \mathrm{DX}^{\wedge} 2-\mathrm{ka}^{*} \mathrm{D} 2+\mathrm{kd} * \mathrm{~L} 2+$ $\mathrm{kn}^{*} \mathrm{~N} 2+\mathrm{SW} 22$

$\mathrm{d}(\mathrm{D} 3) / \mathrm{d}(\mathrm{t})=-\mathrm{U}^{*}(\mathrm{D} 3-\mathrm{D} 2) / \mathrm{DX}+\mathrm{E}^{*}\left(\mathrm{D} 4-2^{*} \mathrm{D} 3+\mathrm{D} 2\right) / \mathrm{DX} \wedge 2-\mathrm{ka}^{*} \mathrm{D} 3+\mathrm{kd} * \mathrm{~L} 3+$ $\mathrm{kn}^{*} \mathrm{~N} 3+\mathrm{SW} 23$

$\mathrm{d}(\mathrm{D} 4) / \mathrm{d}(\mathrm{t})=-\mathrm{U}^{*}(\mathrm{D} 4-\mathrm{D} 3) / \mathrm{DX}+\mathrm{E}^{*}(\mathrm{D} 5-2 * \mathrm{D} 4+\mathrm{D} 3) / \mathrm{DX}^{\wedge} 2-\mathrm{ka}^{*} \mathrm{D} 4+\mathrm{kd} * \mathrm{~L} 4+$ $\mathrm{kn}^{*} \mathrm{~N} 4+\mathrm{SW} 24$

$\mathrm{d}(\mathrm{D} 5) / \mathrm{d}(\mathrm{t})=-\mathrm{U}^{*}(\mathrm{D} 5-\mathrm{D} 4) / \mathrm{DX}+\mathrm{E}^{*}\left(\mathrm{D} 6-2^{*} \mathrm{D} 5+\mathrm{D} 4\right) / \mathrm{DX} \wedge 2-\mathrm{ka}^{*} \mathrm{D} 5+\mathrm{kd} * \mathrm{~L} 5+$ $\mathrm{kn} * \mathrm{~N} 5+\mathrm{SW} 25$

$\mathrm{d}(\mathrm{D} 6) / \mathrm{d}(\mathrm{t})=-\mathrm{U}^{*}(\mathrm{D} 6-\mathrm{D} 5) / \mathrm{DX}+\mathrm{E}^{*}\left(\mathrm{D} 7-2^{*} \mathrm{D} 6+\mathrm{D} 5\right) / \mathrm{DX} \wedge 2-\mathrm{ka}^{*} \mathrm{D} 6+\mathrm{kd} * \mathrm{~L} 6+$ $\mathrm{kn} * \mathrm{~N} 6+\mathrm{SW} 26$

$\mathrm{d}(\mathrm{D} 7) / \mathrm{d}(\mathrm{t})=-\mathrm{U}^{*}(\mathrm{D} 7-\mathrm{D} 6) / \mathrm{DX}+\mathrm{E}^{*}\left(\mathrm{D} 8-2^{*} \mathrm{D} 7+\mathrm{D} 6\right) / \mathrm{DX} \wedge 2-\mathrm{ka}^{*} \mathrm{D} 7+\mathrm{kd} * \mathrm{~L} 7+$ $\mathrm{kn} * \mathrm{~N} 7+\mathrm{SW} 27$

$\mathrm{d}(\mathrm{D} 8) / \mathrm{d}(\mathrm{t})=-\mathrm{U}^{*}(\mathrm{D} 8-\mathrm{D} 7) / \mathrm{DX}+\mathrm{E}^{*}\left(\mathrm{D} 9-2^{*} \mathrm{D} 8+\mathrm{D} 7\right) / \mathrm{DX} \wedge 2-\mathrm{ka}^{*} \mathrm{D} 8+\mathrm{kd} * \mathrm{~L} 8+$ $\mathrm{kn}^{*} \mathrm{~N} 8+\mathrm{SW} 28$

$\mathrm{d}(\mathrm{D} 9) / \mathrm{d}(\mathrm{t})=-\mathrm{U}^{*}(\mathrm{D} 9-\mathrm{D} 8) / \mathrm{DX}+\mathrm{E}^{*}\left(\mathrm{D} 10-2^{*} \mathrm{D} 9+\mathrm{D} 8\right) / \mathrm{DX} \wedge 2-\mathrm{ka}^{*} \mathrm{D} 9+\mathrm{kd} * \mathrm{~L} 9+$ $\mathrm{kn}^{*} \mathrm{~N} 9+\mathrm{SW} 29$

$\mathrm{d}(\mathrm{D} 10) / \mathrm{d}(\mathrm{t})=-\mathrm{U}^{*}(\mathrm{D} 10-\mathrm{D} 9) / \mathrm{DX}+\mathrm{E}^{*}\left(\mathrm{D} 11-2^{*} \mathrm{D} 10+\mathrm{D} 9\right) / \mathrm{DX} \wedge 2-\mathrm{ka}^{*} \mathrm{D} 10+\mathrm{kd} *$ $\mathrm{L} 10+\mathrm{kn} * \mathrm{~N} 10+\mathrm{SW} 210$ 
Tabla 3

Condiciones iniciales del programa P_REG_NO_ESTAC.pol, con ingreso instantáneo de contaminante

\begin{tabular}{cccl}
\hline $\begin{array}{l}\text { Carga orgánica } \\
\text { carbonácea inicial }\end{array}$ & $\begin{array}{l}\text { Carga orgánica } \\
\text { nitrogenada inicial }\end{array}$ & $\begin{array}{l}\text { Déficit inicial de oxigeno } \\
\text { a lo largo del flujo }\end{array}$ & $\begin{array}{l}\text { Concentración de } \\
\text { oxigeno }\end{array}$ \\
\hline L1(0) $=80$ & $\mathrm{~N} 1(0)=20$ & $\mathrm{D} 1(0)=0.2$ & $\mathrm{C} 1=\mathrm{Cs}-\mathrm{D} 1$ \\
$\mathrm{~L} 2(0)=0$ & $\mathrm{~N} 2(0)=0$ & $\mathrm{D} 2(0)=0.2$ & $\mathrm{C} 2=\mathrm{Cs}-\mathrm{D} 2$ \\
$\mathrm{~L} 3(0)=0$ & $\mathrm{~N} 3(0)=0$ & $\mathrm{D} 3(0)=0.2$ & $\mathrm{C} 3=\mathrm{Cs}-\mathrm{D} 3$ \\
$\mathrm{~L} 4(0)=0$ & $\mathrm{~N} 4(0)=0$ & $\mathrm{D} 4(0)=0.2$ & $\mathrm{C} 4=\mathrm{Cs}-\mathrm{D} 4$ \\
$\mathrm{~L} 5(0)=0$ & $\mathrm{~N} 5(0)=0$ & $\mathrm{D} 5(0)=0.2$ & $\mathrm{C} 5=\mathrm{Cs}-\mathrm{D} 5$ \\
$\mathrm{~L} 6(0)=0$ & $\mathrm{~N} 6(0)=0$ & $\mathrm{D} 6(0)=0.2$ & $\mathrm{C6}=\mathrm{Cs}-\mathrm{D} 6$ \\
$\mathrm{~L} 7(0)=0$ & $\mathrm{~N} 7(0)=0$ & $\mathrm{D} 7(0)=0.2$ & $\mathrm{C} 7=\mathrm{Cs}-\mathrm{D} 7$ \\
$\mathrm{~L} 8(0)=0$ & $\mathrm{~N} 8(0)=0$ & $\mathrm{D} 8(0)=0.2$ & $\mathrm{C} 8=\mathrm{C}-\mathrm{D} 8$ \\
$\mathrm{~L} 9(0)=0$ & $\mathrm{~N} 9(0)=0$ & $\mathrm{D} 9(0)=0.2$ & $\mathrm{C} 9=\mathrm{Cs}-\mathrm{D} 9$ \\
$\mathrm{~L} 10(0)=0$ & $\mathrm{~N} 10(0)=0$ & $\mathrm{D} 10(0)=0.2$ & $\mathrm{C} 10=\mathrm{Cs}-\mathrm{D} 10$ \\
$\mathrm{~L} 11=\mathrm{L} 10$ & $\mathrm{~N} 11=\mathrm{N} 10$ & $\mathrm{D} 11=\mathrm{D} 10$ & \\
\hline
\end{tabular}

Tabla 4

Parámetros del programa P_REG_NO_ESTAC.pol, con ingreso instantáneo de contaminante

\begin{tabular}{ll}
\hline Valores de los parámetros & Denominación de los parámetros \\
\hline $\mathrm{U}=2$ & Velocidad del fluido $(\mathrm{m} / \mathrm{s})$ \\
$\mathrm{L} 0=0$ & Carga orgánica carbonácea aguas arriba \\
$\mathrm{N} 0=0$ & Carga orgánica nitrogenada aguas arriba \\
$\mathrm{D} 0=0.2$ & Déficit inicial de oxígeno disuelto $(\mathrm{ppm})$ \\
$\mathrm{DX}=20$ & Incremento longitudinal $(\mathrm{m} / \mathrm{s})$ \\
$\mathrm{ka}=0.20$ & Constante de reaireacion $(1 / \mathrm{d})$ \\
$\mathrm{kd}=0.06$ & Constante de remoción de materia carbonácea por reacción $(1 / \mathrm{d})$ \\
$\mathrm{kn}=0.04$ & Constante de remoción de materia nitrogenada por reacción $(1 / \mathrm{d})$ \\
$\mathrm{kr}=0.08$ & Tasa de remoción de materia carbonácea $(1 / \mathrm{d})$ \\
$\mathrm{Cs}=\mathrm{CSP} 2$ & Concentración de oxígeno saturado $(\mathrm{ppm})$ \\
$\mathrm{E}=0.08$ & Coeficiente de difusión $\left(\mathrm{m}^{2} / \mathrm{s}\right)$ \\
\hline
\end{tabular}

Tabla 5

Cargas puntuales de contaminantes orgánicos y contribuciones difusas de oxigeno del programa $P_{-}$ REG_NO_ESTAC.pol, con ingreso instantáneo de contaminante

\begin{tabular}{lcl}
\hline Carga orgánica carbonácea & Carga orgánica nitrogenada & Fuentes difusas de oxigeno $\left(^{*}\right)$ \\
\hline WC1 $=0.005$ & WN1 $=0.004$ & SW21 $=-0.002$ \\
WC2 $=0.006$ & WN2 $=0.001$ & SW22 $=0.002$ \\
WC3 $=0.002$ & WN3 $=0.002$ & SW23 $=0.002$ \\
WC4 $=0.007$ & WN4 $=0.008$ & SW2 $=0.002$ \\
WC5 $=0.008$ & WN5 $=0.007$ & SW25 $=-0.002$ \\
WC6 $=0.002$ & WN6 $=0.002$ & SW26 $=0.002$ \\
WC7 $=0.004$ & WN7 $=0.004$ & SW27 $=-0.0002$ \\
WC8 $=0.003$ & WN8 $=0.006$ & SW28 $=0.0002$ \\
WC9 $=0.002$ & WN9 $=0.002$ & SW210 $=0.0000$ \\
WC10 $=0.001$ & WN10 $=0.001$ &
\end{tabular}

$\left({ }^{*}\right)$ Fuentes difusas de oxígeno valores positivos para a la fotosíntesis y valores negativos para la respiración 
\# Concentración de saturación de oxígeno en agua pura

$\mathrm{CS} 1=\exp \left(-139.34411+1.575701 \mathrm{E} 5 / \mathrm{TK}-6.642308 \mathrm{E} 7 / \mathrm{TK}^{\wedge} 2+1.2438 \mathrm{E} 10 / \mathrm{TK}^{\wedge} 3-\right.$ 8.621949E11 / TK^4)

\# Concentración de saturación en agua con contenido de sales

$\mathrm{CS} 2=\exp (\ln (\mathrm{CS} 1)-\mathrm{S} *(1.7674 \mathrm{E}-2-1.0754 \mathrm{E} 1 / \mathrm{TK}+2.1407 \mathrm{E} 3 / \mathrm{TK} \wedge 2))$

\# Concentración de saturación en función a la presión

$\mathrm{CSP} 2=\operatorname{CS} 2 * \mathrm{P} *((\mathrm{~A} / \mathrm{B}))$

$\mathrm{A}=(1-\mathrm{PWV} / \mathrm{P}) *\left(1-\mathrm{THETA}^{*} \mathrm{P}\right)$ \# Factor

$\mathrm{B}=(1-\mathrm{PWV}) *(1-$ THETA $) \#$ Factor

\# Presión de vapor del agua

$\mathrm{PWV}=\exp (11.8571-3840.70 / \mathrm{TK}-216.961 / \mathrm{TK} \wedge 2)$

\# Factor de corrección por temperatura

THETA $=0.000975-1.426 \mathrm{E}-5 * \mathrm{~T}+6.436 \mathrm{E}-8 * \mathrm{~T} \wedge 2$

\# Presión atmosférica

$\mathrm{P}=548 / 760$

$\mathrm{T}=20$

$\mathrm{TK}=\mathrm{T}+273.15$

$\mathrm{S}=25$ \# concentración de sal

$\mathrm{t}(0)=0$

$\mathrm{t}(\mathrm{f})=40$

\section{Anexo 2: Programa P_REG_ESTAC.pol}

Sistema de ecuaciones algebraicas lineales para el cálculo de los perfiles de concentración

$$
\begin{aligned}
& \text { \#Demanda carbonácea } \\
& \mathrm{f}(\mathrm{L} 1)=\mathrm{L} 2+\mathrm{A} 1{ }^{*} \mathrm{~L} 1+\mathrm{B} * \mathrm{~L} 0+\mathrm{F} 1 \\
& \mathrm{f}(\mathrm{L} 2)=\mathrm{L} 3+\mathrm{A} 1{ }^{*} \mathrm{~L} 2+\mathrm{B} * \mathrm{~L} 1+\mathrm{F} 2 \\
& \mathrm{f}(\mathrm{L} 3)=\mathrm{L} 4+\mathrm{A} 1{ }^{*} \mathrm{~L} 3+\mathrm{B}^{*} \mathrm{~L} 2+\mathrm{F} 3 \\
& \mathrm{f}(\mathrm{L} 4)=\mathrm{L} 5+\mathrm{A} 1 * \mathrm{~L} 4+\mathrm{B} * \mathrm{~L} 3+\mathrm{F} 4 \\
& \mathrm{f}(\mathrm{L} 5)=\mathrm{L} 6+\mathrm{A} 1 * \mathrm{~L} 5+\mathrm{B} * \mathrm{~L} 4+\mathrm{F} 5 \\
& \mathrm{f}(\mathrm{L} 6)=\mathrm{L} 7+\mathrm{A} 1 * \mathrm{~L} 6+\mathrm{B} * \mathrm{~L} 5+\mathrm{F} 6 \\
& \mathrm{f}(\mathrm{L} 7)=\mathrm{L} 8+\mathrm{A} 1{ }^{*} \mathrm{~L} 7+\mathrm{B}^{*} \mathrm{~L} 6+\mathrm{F} 7 \\
& \mathrm{f}(\mathrm{L} 8)=\mathrm{L} 9+\mathrm{A} 1 * \mathrm{~L} 8+\mathrm{B}^{*} \mathrm{~L} 7+\mathrm{F} 8 \\
& \mathrm{f}(\mathrm{L} 9)=\mathrm{L} 10+\mathrm{A} 1 * \mathrm{~L} 9+\mathrm{B} * \mathrm{~L} 8+\mathrm{F} 9 \\
& \mathrm{f}(\mathrm{L} 10)=\mathrm{L} 11+\mathrm{A} 1{ }^{*} \mathrm{~L} 10+\mathrm{B}^{*} \mathrm{~L} 9+\mathrm{F} 10 \\
& \mathrm{f}(\mathrm{L} 11)=\mathrm{L} 12+\mathrm{A} 1{ }^{*} \mathrm{~L} 11+\mathrm{B}{ }^{*} \mathrm{~L} 10+\mathrm{F} 11 \\
& \mathrm{f}(\mathrm{L} 12)=\mathrm{L} 13+\mathrm{A} 1{ }^{*} \mathrm{~L} 12+\mathrm{B} * \mathrm{~L} 11+\mathrm{F} 12 \\
& \mathrm{f}(\mathrm{L} 13)=\mathrm{L} 14+\mathrm{A} 1{ }^{*} \mathrm{~L} 13+\mathrm{B}^{*} \mathrm{~L} 12+\mathrm{F} 13 \\
& \mathrm{f}(\mathrm{L} 14)=\mathrm{L} 15+\mathrm{A} 1{ }^{*} \mathrm{~L} 14+\mathrm{B}^{*} \mathrm{~L} 13+\mathrm{F} 14 \\
& \mathrm{f}(\mathrm{L} 15)=\mathrm{L} 16+\mathrm{A} 1{ }^{*} \mathrm{~L} 15+\mathrm{B} * \mathrm{~L} 14+\mathrm{F} 15 \\
& \mathrm{f}(\mathrm{L} 16)=\mathrm{L} 17+\mathrm{A} 1{ }^{*} \mathrm{~L} 16+\mathrm{B} * \mathrm{~L} 15+\mathrm{F} 16 \\
& \mathrm{f}(\mathrm{L} 17)=\mathrm{L} 18+\mathrm{A} 1{ }^{*} \mathrm{~L} 17+\mathrm{B} * \mathrm{~L} 16+\mathrm{F} 17 \\
& \mathrm{f}(\mathrm{L} 18)=\mathrm{L} 19+\mathrm{A} 1{ }^{*} \mathrm{~L} 18+\mathrm{B} * \mathrm{~L} 17+\mathrm{F} 18 \\
& \mathrm{f}(\mathrm{L} 19)=\mathrm{L} 20+\mathrm{A} 1{ }^{*} \mathrm{~L} 19+\mathrm{B} * \mathrm{~L} 18+\mathrm{F} 19 \\
& \mathrm{f}(\mathrm{L} 20)=\mathrm{L} 21+\mathrm{A} 1{ }^{*} \mathrm{~L} 20+\mathrm{B} * \mathrm{~L} 19+\mathrm{F} 20
\end{aligned}
$$


\#Demanda nitrogenada

$$
\begin{aligned}
& \mathrm{f}(\mathrm{N} 1)=\mathrm{N} 2+\mathrm{A} 2{ }^{*} \mathrm{~N} 1+\mathrm{B} * \mathrm{~N} 0+\mathrm{G} 1 \\
& \mathrm{f}(\mathrm{N} 2)=\mathrm{N} 3+\mathrm{A} 2{ }^{*} \mathrm{~N} 2+\mathrm{B}^{*} \mathrm{~N} 1+\mathrm{G} 2 \\
& \mathrm{f}(\mathrm{N} 3)=\mathrm{N} 4+\mathrm{A} 2 * \mathrm{~N} 3+\mathrm{B} * \mathrm{~N} 2+\mathrm{G} 3 \\
& \mathrm{f}(\mathrm{N} 4)=\mathrm{N} 5+\mathrm{A} 2 * \mathrm{~N} 4+\mathrm{B} * \mathrm{~N} 3+\mathrm{G} 4 \\
& f(N 5)=N 6+A 2 * N 5+B * N 4+G 5 \\
& \mathrm{f}(\mathrm{N} 6)=\mathrm{N} 7+\mathrm{A} 2 * \mathrm{~N} 6+\mathrm{B}^{*} \mathrm{~N} 5+\mathrm{G} 6 \\
& \mathrm{f}(\mathrm{N} 7)=\mathrm{N} 8+\mathrm{A} 2 * \mathrm{~N} 7+\mathrm{B} * \mathrm{~N} 6+\mathrm{G} 7 \\
& \mathrm{f}(\mathrm{N} 8)=\mathrm{N} 9+\mathrm{A} 2{ }^{*} \mathrm{~N} 8+\mathrm{B}^{*} \mathrm{~N} 7+\mathrm{G} 8 \\
& f(N 9)=\mathrm{N} 10+\mathrm{A} 2 * \mathrm{~N} 9+\mathrm{B} * \mathrm{~N} 8+\mathrm{G} 9 \\
& \mathrm{f}(\mathrm{N} 10)=\mathrm{N} 11+\mathrm{A} 2 * \mathrm{~N} 10+\mathrm{B} * \mathrm{~N} 9+\mathrm{G} 10 \\
& \mathrm{f}(\mathrm{N} 11)=\mathrm{N} 12+\mathrm{A} 2{ }^{*} \mathrm{~N} 11+\mathrm{B}^{*} \mathrm{~N} 10+\mathrm{G} 11 \\
& \mathrm{f}(\mathrm{N} 12)=\mathrm{N} 13+\mathrm{A} 2{ }^{*} \mathrm{~N} 12+\mathrm{B}^{*} \mathrm{~N} 11+\mathrm{G} 12 \\
& \mathrm{f}(\mathrm{N} 13)=\mathrm{N} 14+\mathrm{A} 2{ }^{*} \mathrm{~N} 13+\mathrm{B}^{*} \mathrm{~N} 12+\mathrm{G} 13 \\
& \mathrm{f}(\mathrm{N} 14)=\mathrm{N} 15+\mathrm{A} 2 * \mathrm{~N} 14+\mathrm{B} * \mathrm{~N} 13+\mathrm{G} 14 \\
& \mathrm{f}(\mathrm{N} 15)=\mathrm{N} 16+\mathrm{A} 2 * \mathrm{~N} 15+\mathrm{B} * \mathrm{~N} 14+\mathrm{G} 15 \\
& \mathrm{f}(\mathrm{N} 16)=\mathrm{N} 17+\mathrm{A} 2 * \mathrm{~N} 16+\mathrm{B} * \mathrm{~N} 15+\mathrm{G} 16 \\
& \mathrm{f}(\mathrm{N} 17)=\mathrm{N} 18+\mathrm{A} 2{ }^{*} \mathrm{~N} 17+\mathrm{B} * \mathrm{~N} 16+\mathrm{G} 17 \\
& \mathrm{f}(\mathrm{N} 18)=\mathrm{N} 19+\mathrm{A} 2{ }^{*} \mathrm{~N} 18+\mathrm{B}{ }^{*} \mathrm{~N} 17+\mathrm{G} 18 \\
& \mathrm{f}(\mathrm{N} 19)=\mathrm{N} 20+\mathrm{A} 2{ }^{*} \mathrm{~N} 19+\mathrm{B}{ }^{*} \mathrm{~N} 18+\mathrm{G} 19 \\
& \mathrm{f}(\mathrm{N} 20)=\mathrm{N} 21+\mathrm{A} 2{ }^{*} \mathrm{~N} 20+\mathrm{B} * \mathrm{~N} 19+\mathrm{G} 20
\end{aligned}
$$

\# Déficit de oxigeno

$$
\begin{aligned}
& \mathrm{f}(\mathrm{D} 1)=\mathrm{D} 2+\mathrm{A} 3{ }^{*} \mathrm{D} 1+\mathrm{B}{ }^{*} \mathrm{D} 0+\mathrm{H}^{*} \mathrm{~L} 1+\mathrm{M}^{*} \mathrm{~N} 1+\mathrm{P} 1 \\
& \mathrm{f}(\mathrm{D} 2)=\mathrm{D} 3+\mathrm{A} 3{ }^{*} \mathrm{D} 2+\mathrm{B} * \mathrm{D} 1+\mathrm{H}^{*} \mathrm{~L} 2+\mathrm{M} * \mathrm{~N} 2+\mathrm{P} 2 \\
& \mathrm{f}(\mathrm{D} 3)=\mathrm{D} 4+\mathrm{A} 3^{*} \mathrm{D} 3+\mathrm{B}^{*} \mathrm{D} 2+\mathrm{H}^{*} \mathrm{~L} 3+\mathrm{M}^{*} \mathrm{~N} 3+\mathrm{P} 3 \\
& \mathrm{f}(\mathrm{D} 4)=\mathrm{D} 5+\mathrm{A} 3{ }^{*} \mathrm{D} 4+\mathrm{B}^{*} \mathrm{D} 3+\mathrm{H}^{*} \mathrm{~L} 4+\mathrm{M}^{*} \mathrm{~N} 4+\mathrm{P} 4 \\
& \mathrm{f}(\mathrm{D} 5)=\mathrm{D} 6+\mathrm{A} 3 * \mathrm{D} 5+\mathrm{B} * \mathrm{D} 4+\mathrm{H}^{*} \mathrm{~L} 5+\mathrm{M} * \mathrm{~N} 5+\mathrm{P} 5 \\
& f(D 6)=D 7+A^{*} \mathrm{D} 6+\mathrm{B}^{*} \mathrm{D} 5+\mathrm{H}^{*} \mathrm{~L} 6+\mathrm{M} * \mathrm{~N} 6+\mathrm{P} 6 \\
& \mathrm{f}(\mathrm{D} 7)=\mathrm{D} 8+\mathrm{A} 3{ }^{*} \mathrm{D} 7+\mathrm{B}^{*} \mathrm{D} 6+\mathrm{H}^{*} \mathrm{~L} 7+\mathrm{M}^{*} \mathrm{~N} 7+\mathrm{P} 7 \\
& \mathrm{f}(\mathrm{D} 8)=\mathrm{D} 9+\mathrm{A} 3{ }^{*} \mathrm{D} 8+\mathrm{B} * \mathrm{D} 7+\mathrm{H}^{*} \mathrm{~L} 8+\mathrm{M} * \mathrm{~N} 8+\mathrm{P} 8 \\
& f(D 9)=\mathrm{D} 10+\mathrm{A} 3^{*} \mathrm{D} 9+\mathrm{B}^{*} \mathrm{D} 8+\mathrm{H}^{*} \mathrm{~L} 9+\mathrm{M}^{*} \mathrm{~N} 9+\mathrm{P} 9 \\
& \mathrm{f}(\mathrm{D} 10)=\mathrm{D} 11+\mathrm{A} 3^{*} \mathrm{D} 10+\mathrm{B}^{*} \mathrm{D} 9+\mathrm{H}^{*} \mathrm{~L} 10+\mathrm{M}^{*} \mathrm{~N} 10+\mathrm{P} 10 \\
& \mathrm{f}(\mathrm{D} 11)=\mathrm{D} 12+\mathrm{A} 3{ }^{*} \mathrm{D} 11+\mathrm{B} * \mathrm{D} 10+\mathrm{H}^{*} \mathrm{~L} 11+\mathrm{M}^{*} \mathrm{~N} 11+\mathrm{P} 11 \\
& \mathrm{f}(\mathrm{D} 12)=\mathrm{D} 13+\mathrm{A} 3^{*} \mathrm{D} 12+\mathrm{B} * \mathrm{D} 11+\mathrm{H}^{*} \mathrm{~L} 12+\mathrm{M}^{*} \mathrm{~N} 12+\mathrm{P} 12 \\
& \mathrm{f}(\mathrm{D} 13)=\mathrm{D} 14+\mathrm{A} 3{ }^{*} \mathrm{D} 13+\mathrm{B} * \mathrm{D} 12+\mathrm{H}^{*} \mathrm{~L} 13+\mathrm{M}^{*} \mathrm{~N} 13+\mathrm{P} 13 \\
& \mathrm{f}(\mathrm{D} 14)=\mathrm{D} 15+\mathrm{A} 3^{*} \mathrm{D} 14+\mathrm{B} * \mathrm{D} 13+\mathrm{H}^{*} \mathrm{~L} 14+\mathrm{M}^{*} \mathrm{~N} 14+\mathrm{P} 14 \\
& \mathrm{f}(\mathrm{D} 15)=\mathrm{D} 16+\mathrm{A} 3{ }^{*} \mathrm{D} 15+\mathrm{B} * \mathrm{D} 14+\mathrm{H}^{*} \mathrm{~L} 15+\mathrm{M} * \mathrm{~N} 15+\mathrm{P} 15 \\
& \mathrm{f}(\mathrm{D} 16)=\mathrm{D} 17+\mathrm{A} 3 * \mathrm{D} 16+\mathrm{B} * \mathrm{D} 15+\mathrm{H}^{*} \mathrm{~L} 16+\mathrm{M}^{*} \mathrm{~N} 16+\mathrm{P} 16 \\
& \mathrm{f}(\mathrm{D} 17)=\mathrm{D} 18+\mathrm{A} 3^{*} \mathrm{D} 17+\mathrm{B} * \mathrm{D} 16+\mathrm{H}^{*} \mathrm{~L} 17+\mathrm{M} * \mathrm{~N} 17+\mathrm{P} 17 \\
& \mathrm{f}(\mathrm{D} 18)=\mathrm{D} 19+\mathrm{A} 3{ }^{*} \mathrm{D} 18+\mathrm{B} * \mathrm{D} 17+\mathrm{H}^{*} \mathrm{~L} 18+\mathrm{M}^{*} \mathrm{~N} 18+\mathrm{P} 18 \\
& f(D 19)=D 20+A 3 * D 19+B * D 18+H^{*} \mathrm{~L} 19+\mathrm{M}^{*} \mathrm{~N} 19+\mathrm{P} 19 \\
& \mathrm{f}(\mathrm{D} 20)=\mathrm{D} 21+\mathrm{A} 3^{*} \mathrm{D} 20+\mathrm{B} * \mathrm{D} 19+\mathrm{H}^{*} \mathrm{~L} 20+\mathrm{M}^{*} \mathrm{~N} 20+\mathrm{P} 20
\end{aligned}
$$


$\mathrm{U}=2$ \# Velocidad del fluido $(\mathrm{m} / \mathrm{s})$

L0 $=80$ \# Carga orgánica carbonácea aguas arriba

N0 $=20$ \# Carga orgánica nitrogenada aguas arriba

D0 $=0.2$ \# Déficit inicial de oxigeno disuelto

$\mathrm{DX}=10$ \# Incremento longitudinal

$\mathrm{ka}=0.20$ \# Constante de reaireacion

$\mathrm{kd}=0.06$ \# Constante de remoción de materia carbonácea por reacción

$\mathrm{kn}=0.04$ \# Constante de remoción de materia nitrogenada por reacción

$\mathrm{kr}=0.08$ \# Tasa de remoción de materia carbonácea

$\mathrm{krn}=0.04$ \# Tasa de remoción de materia nitrogenada

Cs $=$ CSP2 \# Cs = 8.9 \# Concentración de oxigeno saturado

$\mathrm{L} 21=\mathrm{L} 20$

$\mathrm{N} 21=\mathrm{N} 20$

$\mathrm{D} 21=\mathrm{D} 20$

$\mathrm{E}=0.08$ \# Coeficiente de difusión turbulenta

$\mathrm{CS} 1=\exp (-139.34411+1.575701 \mathrm{E} 5 / \mathrm{TK}-6.642308 \mathrm{E} 7 / \mathrm{TK} \wedge 2+1.2438 \mathrm{E} 10 / \mathrm{TK} \wedge 3-$ 8.621949E11 / TK^4)

$\mathrm{CS} 2=\exp \left(\ln (\mathrm{CS} 1)-\mathrm{S}^{*}(1.7674 \mathrm{E}-2-1.0754 \mathrm{E} 1 / \mathrm{TK}+2.1407 \mathrm{E} 3 / \mathrm{TK} \wedge 2)\right)$

\# Revisar esta formula

$\mathrm{CSP} 2=\mathrm{CS} 2 * \mathrm{P} *((\mathrm{AA} / \mathrm{BB}))$

$\mathrm{AA}=(1-\mathrm{PWV} / \mathrm{P}) *\left(1-\mathrm{THETA}^{*} \mathrm{P}\right)$

$\mathrm{BB}=(1-\mathrm{PWV}) *(1-\mathrm{THETA})$

$\mathrm{PWV}=\exp (11.8571-3840.70 / \mathrm{TK}-216.961 / \mathrm{TK} \wedge 2)$

$\mathrm{THETA}=0.000975-1.426 \mathrm{E}-5 * \mathrm{~T}+6.436 \mathrm{E}-8 * \mathrm{~T} \wedge 2$

$\mathrm{P}=548 / 760$

$\mathrm{T}=20$

$\mathrm{TK}=\mathrm{T}+273.15$

$S=25$ \# concentración de sal

\#Parámetros del cambio de variables

$\mathrm{A} 1=-2-\mathrm{U}^{*} \mathrm{DX} / \mathrm{E}-\mathrm{DX}^{\wedge} 2^{*} \mathrm{kr} / \mathrm{E}$

$\mathrm{A} 2=-2-\mathrm{U}^{*} \mathrm{DX} / \mathrm{E}-\mathrm{DX}^{\wedge} 2^{*} \mathrm{krn} / \mathrm{E}$

$\mathrm{A} 3=-2-\mathrm{U}^{*} \mathrm{DX} / \mathrm{E}-\mathrm{DX}^{\wedge} 2 * \mathrm{ka} / \mathrm{E}$

$\mathrm{B}=\mathrm{U}^{*} \mathrm{DX} / \mathrm{E}$

$\mathrm{H}=\mathrm{DX} \wedge 2^{*} \mathrm{kd} / \mathrm{E}$

$\mathrm{M}=\mathrm{DX}^{\wedge} 2 * \mathrm{kn} / \mathrm{E}$ 
Tabla 6

Cargas puntuales de materia orgánica y contribuciones difusas de oxigeno del programa $P_{-} R E G_{-}$ ESTAC.pol, con ingreso instantáneo de contaminante.

\begin{tabular}{ccl}
\hline Carga orgánica carbonácea & Carga orgánica nitrogenada & Fuentes difusas \\
\hline WC1 $=0.005$ & WN1 $=0.004$ & SW21 $=-0.002$ \\
WC2 $=0.006$ & WN2 $=0.001$ & SW22 $=0.002$ \\
WC3 $=0.002$ & WN3 $=0.002$ & SW23 $=0.002$ \\
WC4 $=0.007$ & WN4 $=0.008$ & SW24 $=0.002$ \\
WC5 $=0.008$ & WN5 $=0.007$ & SW25 $=-0.002$ \\
WC6 $=0.002$ & WN6 $=0.002$ & SW26 $=0.002$ \\
WC7 $=0.004$ & WN7 $=0.004$ & SW27 $=-0.0002$ \\
WC8 $=0.003$ & WN8 $=0.006$ & SW29 $=0.002$ \\
WC9 $=0.002$ & WN9 $=0.002$ & SW210 $=0.0001$ \\
WC10 $=0.001$ & WN10 $=0.001$ & SW211 $=-0.002$ \\
WC1 $=0.005$ & WN11 $=0.004$ & SW212 $=0.002$ \\
WC1 $=0.006$ & WN12 $=0.001$ & SW213 $14=0.002$ \\
WC13 $=0.002$ & WN13 $=0.002$ & SW215 $=-0.002$ \\
WC14 $=0.007$ & WN14 $=0.008$ & SW216 $=0.002$ \\
WC1 $=0.008$ & WN15 $=0.007$ & SW217 $=-0.0002$ \\
WC16 $=0.002$ & WN16 $=0.002$ & SW218 $=0.0002$ \\
WC17 $=0.004$ & WN17 $=0.004$ & SW219 $=0.002$ \\
WC1 $=0.003$ & WN18 $=0.006$ & SW220 $=0.0001$ \\
WC19 $=0.002$ & WN19 $=0.002$ &
\end{tabular}

\section{Nomenclatura:}

$L_{C}$ : demanda bioquímica de oxígeno carbonosa remanente $(\mathrm{mg} / \mathrm{L})$

$L_{N}$ : demanda bioquímica de oxígeno nitrogenada remanente $(\mathrm{mg} / \mathrm{L})$

$D: \quad$ Déficit de oxígeno ( ), $(\mathrm{mg} / \mathrm{L})$

$W_{L}{ }^{1}$ : Carga contaminante de DBO carbonosa, $(\mathrm{mg} / \mathrm{L} / \mathrm{h})$

$W_{N}{ }^{1}$ : Carga contaminante de DBO nitrogenada, $(\mathrm{mg} / \mathrm{L} / \mathrm{h})$

$\sum W_{2}$ : Fuentes y sumideros de oxígeno (fotosíntesis, respiración, sedimentación), etc. (mg/ $\mathrm{L} / \mathrm{h})$.

$k_{r}$ : Tasa de remoción de la demanda bioquímica de oxígeno carbonosa (d-1)

$k_{r n}:$ Tasa de remoción de la demanda bioquímica de oxígeno nitrogenada (d-1)

$k_{a}: \quad$ Tasa de reaireación, $(\mathrm{d}-1)$

$k_{d}$ : Tasa de desoxigenación carbonácea, (d-1)

$k_{n}$ : Tasa de nitrificación, (d-1)

$E$ : Coeficiente de dispersión longitudinal $(\mathrm{m} 2 / \mathrm{s})$ 


\section{Referencias}

Autoridad Nacional del Agua, ANA (2017). Guía para la determinación de la zona de mezcla y la evaluación del impacto del vertimiento de aguas residuales tratadas a un cuerpo natural de agua. ANA, Lima. Disponible en: https://hdl.handle. net/20.500.12543/900

Anguiano, V. J. (2012). Determinación del coeficiente de reaireación en un tramo corto de un río caudaloso con flujo turbulento, mediante el uso de los trazadores hexafluoruros de azufre (SF6) y Rodamina WT. Facultad de Ingeniería Química, Universidad Michoacana de San Nicolás de Hidalgo. México.

Amarilla, R., (2017). Modelamiento matemático del contenido de oxígeno disuelto en el brazo ańa cua, aguas abajo de la represa de Yacireta. Tesis de maestría en ecohidrología, Universidad de Buenos Aires, Argentina.

Benítez Rodas, G.A. (2013). Análisis y modelización de la inactivación de escherichia coli en aguas residuales. http://mail.conacyt.gov.py/ proniii/2955172.pdf

Boluda B., (2011) "Modelización de Vertidos de Aguas Residuales en Sistemas Fluviales". Departamento de Ingeniería Química, Universidad de Alicante. Francia.

Brenner, A., Shacham, M. \& Cutlip, M. (2005). Applications of mathematical software packages for modelling and simulations in environmental engineering education.

Cajas, V., (2015). Estimación de valores de contaminación difusa para el río Tomebamba en zonas periféricas y urbanas - primera aproximación.

Cárdenas, P., (2016). Propuesta y análisis de un modelo matemático para la determinación de la calidad del agua de aguas superficiales. Universidad Central "Marta Abreu" de Las Villas, Facultad Matemática Física Computación. Cuba.

Chapra, S. (1997). Surface Water Quality Modeling, Waveland Press. Inc. U.S.A.

González-López, R \& Ramírez-León, H. (2011). Modelación numérica de la hidrodinámica, del oxígeno disuelto y la demanda bioquímica de oxígeno en sistemas con vegetación.

Orduz, L. (2016). Determinación y comparación de la tasa de reaireación de los ríos molinos, torca, arzobispo y de la quebrada La Vieja, en la ciudad de Bogotá mediante la aplicación de ecuaciones empíricas. Facultad de Ingeniería Ambiental- Universidad Santo Tomas, Colombia.

Pérez, N. (2017). “Simulación Matemática de la Interacción entre la Demanda Bioquímica de Oxígeno (DBO) y el Oxígeno Disuelto (OD) en el río Chili con el Método de los Elementos Finitos". Universidad Nacional de San Agustín Facultad de Ingeniería de Procesos. Arequipa - Perú. 
Potter, M. \& Wiggert, D. (2002). Mecánica de Fluidos; Edit. Thomson, Tercera Edic., México.

Regalado, M., (2008). Temas de Ciencia y Tecnología, Vol. 12, Nro. 35, México

Zhen-Gang, J. (2008). Hydrodynamics and water quality: modeling rivers, lakes and estuaries, New Jersey, Estados Unidos, John Wiley and Sons, Inc.
Zouiten,H.(2012).Tesis Doctoral:Análisis mediante modelado avanzado de procesos de eutrofización en lagunas litorales: Aplicación a masas de aguas atlánticas y mediterráneas, Universidad de Cantabria, Santander, España.

Zúñiga, T. (2014). Modelación de la calidad de agua del río Malacatos, tramo comprendido desde el Sector Dos Puentes hasta Sauces Norte. Universidad Técnica Particular de Loja, Ingeniería Civil. Loja Ecuador 